\title{
Channel Fading Effect Analysis on Diffusion Cooperation Strategies over Adaptive Networks
}

\author{
Jie Yang ${ }^{1}$, Ehsan Mostafapour ${ }^{2}$, Amir Aminfar ${ }^{2}$, Jie Wang ${ }^{1}$, Hao Huang ${ }^{1}$, Afsaneh Akhbari ${ }^{3}$, \\ C. Ghobadi ${ }^{2}$, Guan Gui ${ }^{{ }^{*}}$ \\ ${ }^{1}$ College of Telecom and Information Engineering, Nanjing University of Posts and Telecom, Nanjing, China \\ ${ }^{2}$ Department of Electrical Engineering Urmia University, Urmia, Iran \\ ${ }^{3}$ Department of Electrical Engineering, Ghiaseddin Jamshid Kashani University, Abyek, Iran. \\ Emails: jyang@njupt.edu.cn, e.mostafapour@urmia.ac.ir, 13675171572@163.com,673496228@qq.com, \\ afakhbari@gmail.com, a.aminfar@urmia.ac.ir, ch.ghobadi@urmia.ac.ir, guiguan@njupt.edu.cn. \\ *Corresponding author: guiguan@njupt.edu.cn
}

Received August 23, 2018; revised November 5, 2018; accepted September 27, 2017; published January 31, 2019

\begin{abstract}
In this paper, we investigate the performance of the diffusion adaptation strategies for parameter estimation in wireless adaptive networks, where the nodes exchange information over noisy and fading wireless channels. This paper shows the differences between the effect of Rayleigh and Rician fading over wireless adaptive networks and proves that the Rician fading is a more practical model in such kinds of networks. Simulation results imply that the effect of Rayleigh fading is more degrading for the estimation process than Rician fading. Also, the simulation results show the performance of adapt then combine (ATC) diffusion algorithm is better than the combine then adapt (CTA) algorithm by merely considering noise in wireless channels. While the performance of CTA prevails ATC over the wireless adaptive network in the presence of noise plus channel fading.
\end{abstract}

Keywords: Adaptive distributed networks, diffusion strategy, fading coefficients, least mean square (LMS) algorithm, Rician model.

This research was supported by National Natural Science Foundation of China grants (No. 61401069), Jiangsu Specially Appointed Professor Grant (RK002STP16001), High-level talent startup grant of Nanjing University of Posts and Telecommunications (XK0010915026) and “1311 Talent Plan” of Nanjing University of Posts and Telecommunications. 


\section{Introduction}

The adaptive networks work with several agents distributed in an area gathering data in order to estimate a desired entity [1-7]. All of these agents have access to some measurements and with the help of their processing unit, they perform local estimations. These estimations are usually performed by simple adaptive algorithms such as least mean square (LMS) in order to reduce processing power. But despite these simple algorithms, the use of complicated cooperation schemes makes adaptive networks a powerful tool for distributed estimation. Several cooperation techniques are presented for the collaboration of agents, but the most useful ones are incremental and diffusion strategies [2].

Diffusion strategy is a more practical mode of cooperation because finding a Hamiltonian cycle for the network topology of the incremental mode is sometimes not possible [5]. Recently, the diffusion strategy has been used in several applications including sparse system identification [6] and multitask networks [8] where the adaptive network is assigned to perform estimations for more than one desired variable.

Although the performances of adaptive networks have been considered in several different environmental conditions like: noisy links ([5], [15] and [16]), imperfect links ([3], [5]) and fading channels ([1], [2] and [4]), we can still find unchecked scenarios in this problem. Also, some new challenges are continually being presented for wireless channels in future wireless communication systems [11-13]. The performance of the incremental strategy in Rayleigh fading condition has been considered in [1], while the authors of [2] have considered the performance in the same Rayleigh channel for diffusion strategy. However, in [2] the exact impact of fading channel on the adaptive network has not been considered directly. Instead, the authors have presumed that the channel estimation is available in sensor nodes and equalization is possible in order to delete the effect of fading. In [14] the use of adaptive networks in channel estimation has been considered. However, in [14] the effect and estimation of Rayleigh fading have only been analyzed with Incremental strategy.

Based on previous studies, in this paper, we examine the direct effect of fading channels on the performance of diffusion adaptation strategy. Besides the Rayleigh fading model, we also consider the scenario of Rician channel. The assumption of Rician model for links among nodes is a more practical one than the Rayleigh fading model because, in this model, we can usually consider a powerful link between adjacent nodes. In this case, we can expect that the performance of networks becomes much better than the previously presented results in [1] and [4]. Our analysis showed that unlike other cases, the fading effect can be more reduced by CTA algorithm and in this case, the performance of the diffusion CTA algorithm is superior to the performance of ATC diffusion algorithm. Also, we showed that the performance of both diffusion schemes in Rician fading is better than Rayleigh fading because in Rician case, as we mentioned, there is a powerful line of sight between the transmitter and the receiver nodes and this phenomenon results in a more accurate data communication between them. The rest of this paper is designed as follows: In part II, we introduce the adaptive model and problem statement. Furthermore, the details of the impact of the fading channels on the diffusion algorithms are presented in this part. In section III. We reviewed the fading models that are used in this paper. In part IV, we presented the simulation results to investigate the channel effects. And in part V, we concluded our paper and suggested further insights. 
Notation: We utilized small bold-faced letters to show vectors and capital bold-faced letters for matrices. The operator $[\cdot]^{*}$ shows complex conjugate for scalar values and Hermitian transposition for matrices. Furthermore, $\mathbb{E}[\cdot]$ is used for presenting the mathematical expectation and the operator $\|\cdot\|$ is utilized for showing the Euclidian norm of a vector.

\section{Problem statement}

Usually, for adaptive networks we assume a linear relation linking the desired values and the input data vectors[1]:

$$
d_{k}(i)=\boldsymbol{u}_{k, i} \boldsymbol{w}^{o}+v_{k}(i)
$$

where $d_{k}(i)$ represents the desired values, $\boldsymbol{u}_{k, i}$ represents the regressor vectors, and $v_{k}(i)$ shows the measurement noise that follows Gaussian distribution. Our purpose is to estimate the optimal weight vector $\boldsymbol{w}^{o}$ by minimizing the global cost:

$$
J^{g l o b}(\boldsymbol{w})=\sum_{k=1}^{N} \mathbb{E}\left|d_{k}(i)-\boldsymbol{u}_{k, i} \boldsymbol{w}\right|^{2}
$$

By minimizing this cost function with respect to $\boldsymbol{w}$ we seek to achieve the optimum weight vector as [7]:

$$
\boldsymbol{w}^{o}=\left(\sum_{k=1}^{N} \boldsymbol{R}_{u, k}\right)^{-1}\left(\sum_{k=1}^{N} \boldsymbol{R}_{d u, k}\right)
$$

where $\boldsymbol{R}_{u, k}=\mathbb{E}\left[\boldsymbol{u}_{k, i}^{*} \boldsymbol{u}_{k, i}\right]$ and $\boldsymbol{R}_{d u, k}=\mathbb{E}\left[d_{k}(i) \boldsymbol{u}_{k, i}^{*}\right]$ represent the correlation of inputs (or regressors) and the cross-correlation of inputs and desired values. Due to the fact that we do not have these correlation quantities, we must use the iterative or adaptive schemes to arrive at the results. Up until now, many centralized and distributed adaptive algorithms have been presented to solve this problem [7, 9]. In distributed algorithms, each sensor node collects data and applies local estimations of the unknown vector namely $\boldsymbol{\psi}_{k}^{(i)}$, then sends this estimation to its neighboring nodes. However, through this sharing of information, the neighboring nodes receive these local estimations with some deficiencies. These deficiencies may occur in the shape of channel noise or fading. In Fig. 1, the additive noise signals and channel coefficients are present in all the links between nodes.

The difference between incremental and diffusion distributed strategies is in this data sharing process. In the incremental strategy, each node only needs one local estimation of its immediate neighboring node to perform its own estimation. But, in the diffusion strategy, each node collects several local estimations from neighboring nodes and by combining them, performs estimation. This shows that the diffusion strategy is more prone to the channel deficiencies. The performance of these strategies must be evaluated and compared in different scenarios.

For assessing the diffusion network estimation performance, two main error criteria are proposed in the literature namely: the Excess Mean Square Error (EMSE) and the Mean Square Deviation (MSD) [1,7]. These values can be obtained by the definition of the following error vectors:

$$
\widetilde{\boldsymbol{\psi}}_{k}^{(i)} \triangleq \boldsymbol{w}^{o}-\boldsymbol{\psi}_{k}^{(i)}
$$




$$
\boldsymbol{e}_{a, k}(i) \triangleq \boldsymbol{u}_{k, i} \widetilde{\boldsymbol{\psi}}_{k-1}^{(i)}
$$

where $\widetilde{\boldsymbol{\psi}}_{k}^{(i)}$ represents the weight error vector. Also, $\boldsymbol{e}_{a, k}(i)$ denotes the apriori local error at node $k$ and iteration $i$. The values of MSD and EMSE in the steady state condition are then:

$$
\begin{gathered}
M S D \triangleq \mathbb{E}\left\|\widetilde{\boldsymbol{\psi}}_{k}^{(\infty)}\right\|^{2} \\
E M S E \triangleq \mathbb{E}\left|\boldsymbol{e}_{a, k}(\infty)\right|^{2}
\end{gathered}
$$

We only need the MSD values for presenting our simulation results. As mentioned, in the diffusion strategy each node needs to combine several local estimations of its neighboring nodes and then perform its own estimation. This process entails two different methods described as below:

\subsection{The Combine Then Adapt (CTA) diffusion strategy with noisy and fading links}

For the CTA strategy first of all the network nodes combine their estimated and sent data and then update their local estimation. We must take heed to the point that the combined estimates that are received estimations from neighboring nodes are affected by channel noise and fading coefficients, through the links. Therefore, we have:

$$
\boldsymbol{t}_{k, l}^{(i)}=h_{k, l}^{(i)} \boldsymbol{\psi}_{k}^{(i)}+\boldsymbol{q}_{k, l}^{(i)}, \quad l \in \mathcal{N}_{k}
$$

where $\boldsymbol{t}_{k, l}^{(i)}$ represents the received estimations from node $l$ to node $k, h_{k, l}^{(i)}$ is the channel coefficient among these nodes at each iteration $i$ and $\boldsymbol{q}_{k, l}^{(i)}$ is the channel noise that follows Gaussian distribution with zero mean and the covariance matrix of $\boldsymbol{Q}_{k, l}=\mathbb{E}\left[\boldsymbol{q}_{k, l}^{(i)} \boldsymbol{q}_{k, l}^{(i) *}\right]$. As we can see in Fig. 1, the channel coefficients $\left(h_{k, l}^{(i)}\right)$ are multiplied to the exchanged data and channel noises $\left(\boldsymbol{q}_{k, l}^{(i)}\right)$ are added to them.

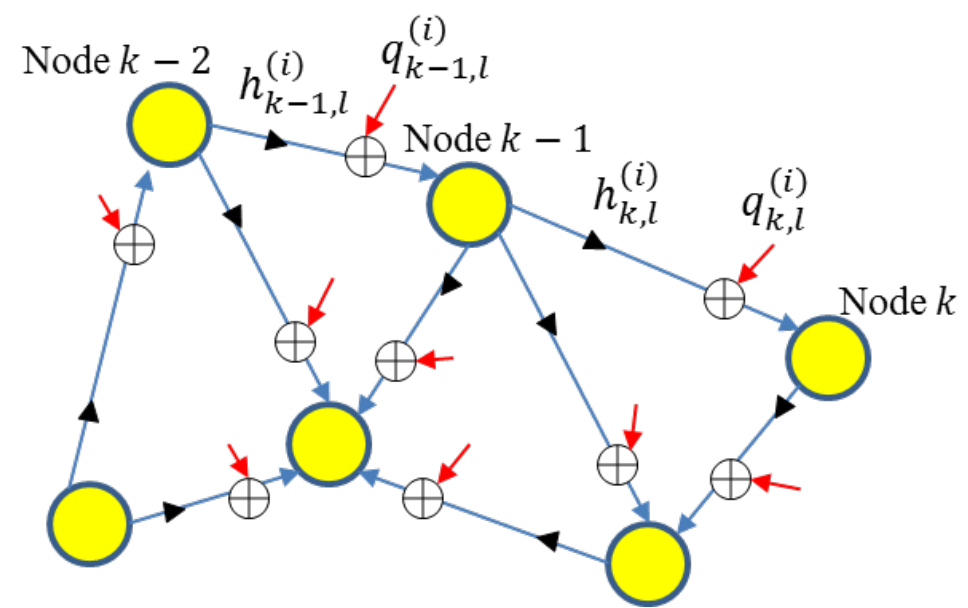

Fig. 1. The diffusion adaptive network with fading and noisy channels. 
The CTA algorithm formulation with the presence of the channel fading and noise is then given as:

$$
\begin{gathered}
\boldsymbol{\phi}_{k}^{(i-1)}=\sum_{l \in \mathcal{N}_{k}} a_{k, l} \boldsymbol{t}_{k, l}^{(i-1)} \\
\boldsymbol{\psi}_{k}^{(i)}=\boldsymbol{\phi}_{k}^{(i-1)}+\mu_{k} \boldsymbol{u}_{k, i}^{*}\left(d_{k}(i)-\boldsymbol{u}_{k, i} \boldsymbol{\phi}_{k}^{(i-1)}\right)
\end{gathered}
$$

where $a_{k, l}$ represent the combination coefficients that play an important role in the performance of the diffusion network. There are several techniques to choose these values that are described in [7]. In this paper, we used the Uniform combination method for determining these coefficients. In this method we have:

$$
a_{l, k}=\left\{\begin{array}{lr}
\frac{1}{n_{k}}, & l \in \mathcal{N}_{k} \\
0, & \text { otherwise }
\end{array}\right.
$$

Where $n_{k} \triangleq\left|\mathcal{N}_{k}\right|$ represents the size of the neighborhood of node $k$ that can be 2 to 7 . All of the neighbors of node $k$ are assigned uniformly with the same weights, $\frac{1}{n_{k}}$.

\subsection{Adapt Then Combine (ATC) diffusion strategy with noisy and fading links}

The next diffusion algorithm is the ATC where the network nodes first update their local estimations and then combine their estimations with the neighboring nodes:

$$
\boldsymbol{\phi}_{k}^{(i)}=\boldsymbol{\psi}_{k}^{(i-1)}+\mu_{k} \boldsymbol{u}_{k, i}^{*}\left(d_{k}(i)-\boldsymbol{u}_{k, i} \boldsymbol{\psi}_{k}^{(i-1)}\right)
$$

For the CTA algorithm, we used the $\boldsymbol{r}_{k, l}^{(i)}$ notation to represent the received information from node $l$ to node $k$ and can write

$$
\begin{gathered}
\boldsymbol{r}_{k, l}^{(i)}=h_{k, l}^{(i)} \boldsymbol{\phi}_{k}^{(i)}+\boldsymbol{q}_{k, l}^{(i)}, \quad l \in \mathcal{N}_{k} \\
\boldsymbol{\psi}_{k}^{(i)}=\sum_{l \in \mathcal{N}_{k}} a_{k, l} \boldsymbol{r}_{k, l}^{(i)}
\end{gathered}
$$

Again, we used the Uniform combination method for determining the $a_{k, l}$ values in this algorithm.

\section{Overview of fading channel models}

To the best of our knowledge, the effects of the no wireless radio fading models other than the Rayleigh distribution have been considered in the performance of the adaptive networks [1]-[4], while it is a very important issue. In this paper, we studied the exact impact of fading channel models on the performance of diffusion adaptation strategies, here we consider two main fading channel models. In order to simulate the fading condition, we can produce the channel coefficients between each pair of nodes $l$ and $k$ separately according to the presumed model. 


\subsection{Rayleigh fading channel model}

The fading channel coefficients can be produced by Rician or Rayleigh distributions, based on the existence or the absence of the specular signal component or the line of sight [10-11]. Fading can be modeled as Rayleigh if the multiple reflective paths are many and there is no main line-of-sight (LOS) path between the transmitter and the receiver. The fading coefficient $h_{i}$ at the $i$ th time instant can be given as the combination of two Gaussian variables:

$$
h_{k, l}^{(i)}=\sqrt{\left(x_{k, l}^{(i)}\right)^{2}+\left(y_{k, l}^{(i)}\right)^{2}}
$$

where $x_{k, l}^{(i)}$ and $y_{k, l}^{(i)}$ represent two independent zero mean Gaussian variables with variance $\sigma_{0}^{2}$ and in this case the Rayleigh distribution of channel envelop is given as:

$$
f_{\text {Rayleigh }}(h)=\frac{h}{\sigma_{0}^{2}} \exp \left[-\frac{\left(h^{2}\right)}{2 \sigma_{0}^{2}}\right] \quad, r \geq 0
$$

\subsection{Rician fading channel model}

In [10-11], it is mentioned that if there is a main LOS path in the communication link between transmitter and receiver, then the fading can be modeled as Rician. The fading coefficient $h_{k, l}^{(i)}$ at the $i$ th time instant can be given as:

$$
h_{k, l}^{(i)}=\sqrt{\left(x_{k, l}^{(i)}+\beta\right)^{2}+\left(y_{k, l}^{(i)}\right)^{2}}
$$

where $\beta$ is the amplitude of the specular component and we define the Rician $K$ factor (not to be mistaken with $k$ the node indices) as:

$$
K=\frac{\beta^{2}}{2 \sigma_{0}^{2}}
$$

that shows the ratio of the specular to the diffuse energy, and changing its values has a significant impact on the performance of the network. In this case, the Rician distribution of channel envelop is given by:

$$
f_{\text {Rice }}(h)=\frac{h}{\sigma_{0}^{2}} \exp \left[-\frac{\left(h^{2}+\beta^{2}\right)}{2 \sigma_{0}^{2}}\right] I_{0}\left[\frac{h \beta}{\sigma_{0}^{2}}\right], r \geq 0
$$

where $I_{0}[$.$] is the zeroth-order modified Bessel function [11] which is shown in Fig. 2$. 


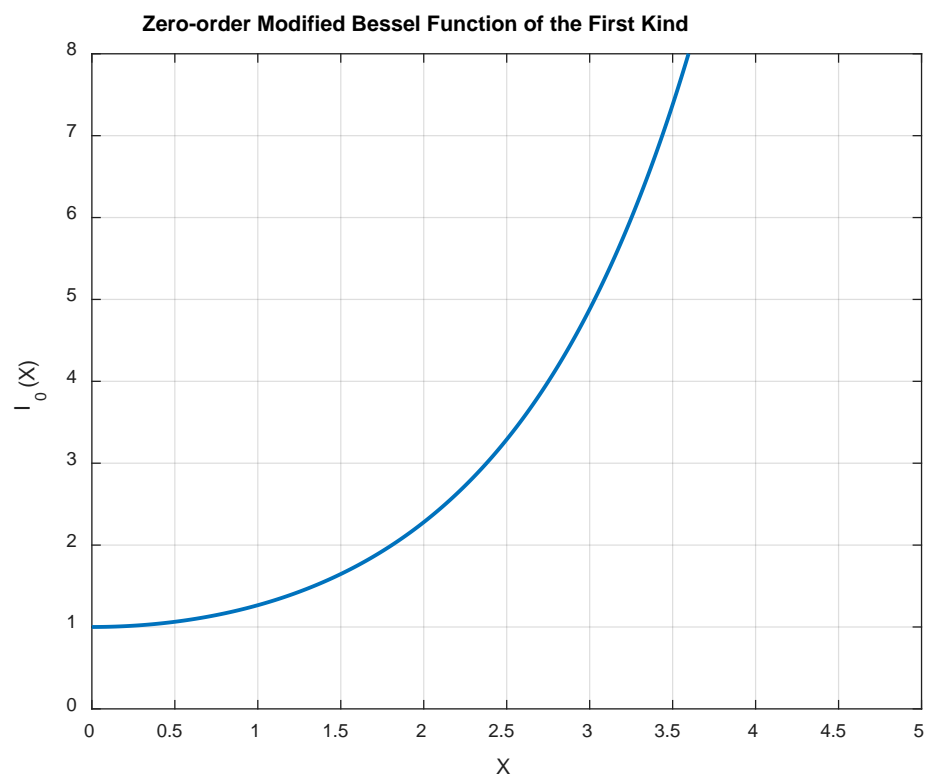

Fig. 2. The zeroth-order modified Bessel function.

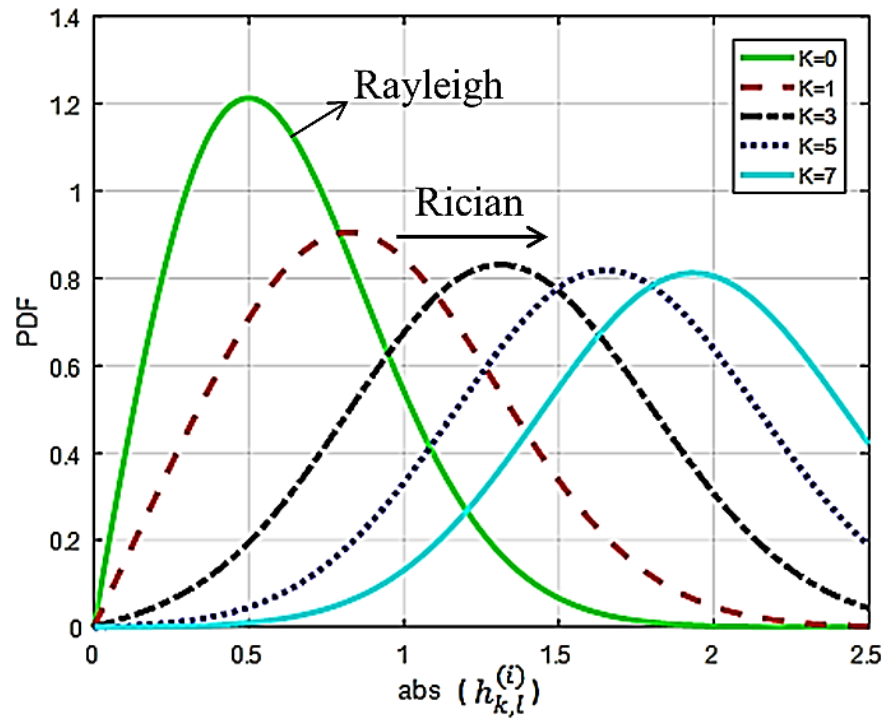

Fig. 3. The PDFs of Rayleigh and Rician distributions based on the Rician $K$ factor.

Following the relations of (16) and (19), the difference between the Rayleigh and the Rician PDFs are shown in Fig. 3.

\section{Simulation studies}

After the consideration of the proposed channel fading models, here are presented the impacts of the Rayleigh and Rician channel models on the performance diffusion ATC and CTA algorithms. We designed a network with 20 nodes with the structure of Fig. 4. 


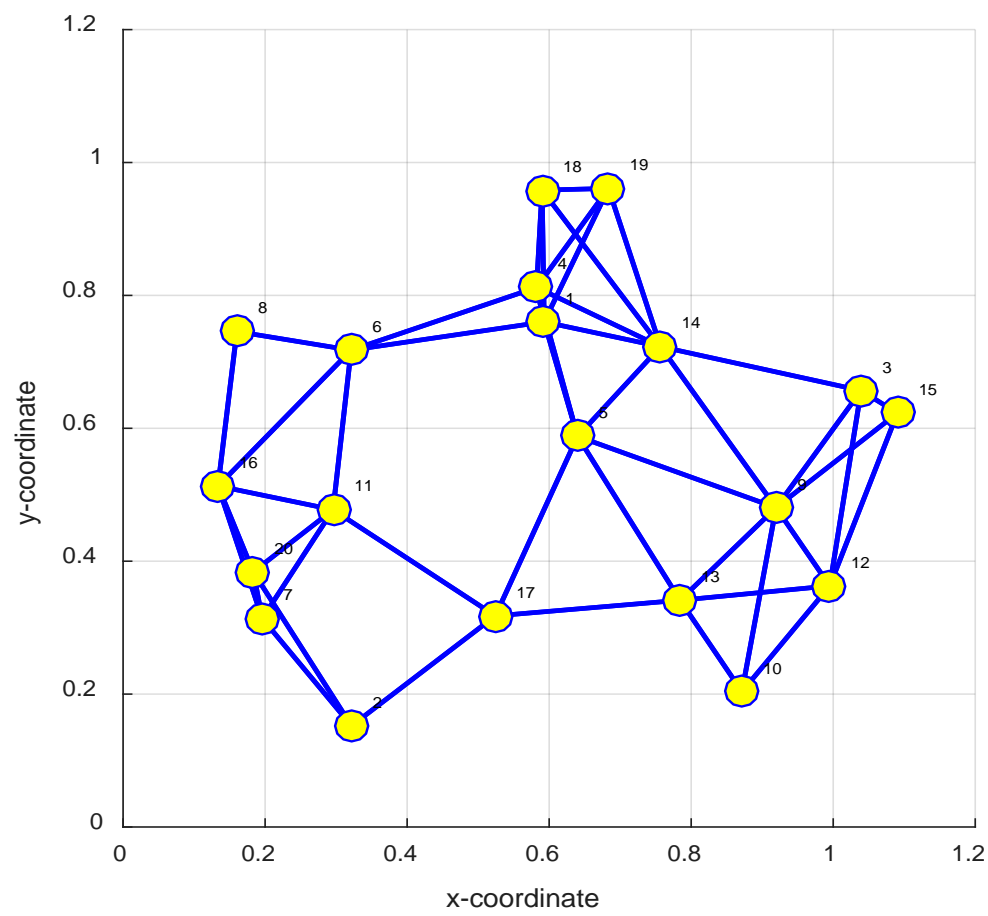

Fig. 4. The structure of a diffusion adaptive network with 20 nodes.
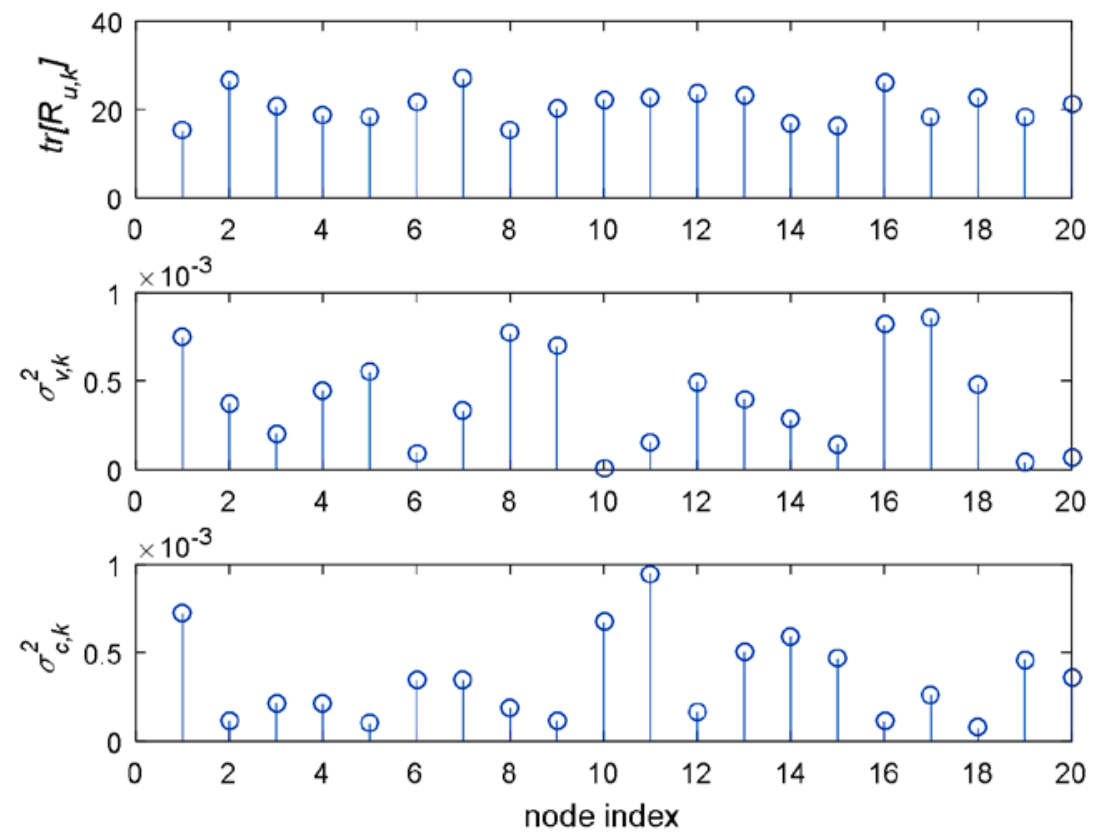

Fig. 5. The statistical values of the utilized parameters. 
For the topology of Fig. 4, the amount of neighboring nodes for each agent is different and it can be between 2 and 7 nodes. For the combination coefficients $a_{k, l}$ we specified them due to these number of neighboring nodes. Our goal is to estimate the unknown optimal vector with the size of $M=4$ like $\boldsymbol{w}^{o}=\left[\begin{array}{llll}1 & 1 & 1 & 1\end{array}\right]^{T} / \sqrt{4}$. The detailed features of the utilized variables in our simulations are given in Fig. 5.

In Fig. 6, we presumed that the variance of the measurement noise is equal to $\sigma_{v, k}^{2}$ for each node $k$ and the covariance matrix of channel noise between the node $k, l$ is equivalent to $\boldsymbol{Q}_{k, l}=\sigma_{c, k}^{2} \boldsymbol{I}$, where $\boldsymbol{I}$ is the $M \times M$ identity matrix, and therefore we only need to know the variance values. For generating our fading channel coefficients as in (15) and (17) we used zero mean Gaussian variables with variance $\sigma_{0}^{2}=1$. In this case, if we consider $K$ for three values of 0,5 and 7 , the $\beta$ becomes $0,3.16$ and 3.74 respectively.

First, we compare the performance of the network in a noisy environment and a noisy plus fading condition using MSD criteria. Also, in Fig. 6, we presented that the performance of the network in a noisy environment is much better than its performance in a fading environment. In all of the simulations, the performances of Both ATC and CTA algorithms are presented. The important fact is that the performance of CTA diffusion strategy is more acceptable than ATC diffusion when we have fading. We assume that this is because of the combination method of the CTA diffusion algorithm that performs adaptation process after the combination, and this allows the adaptive algorithm in each sensor to reduce some of the effects of fading in the adaptation step.

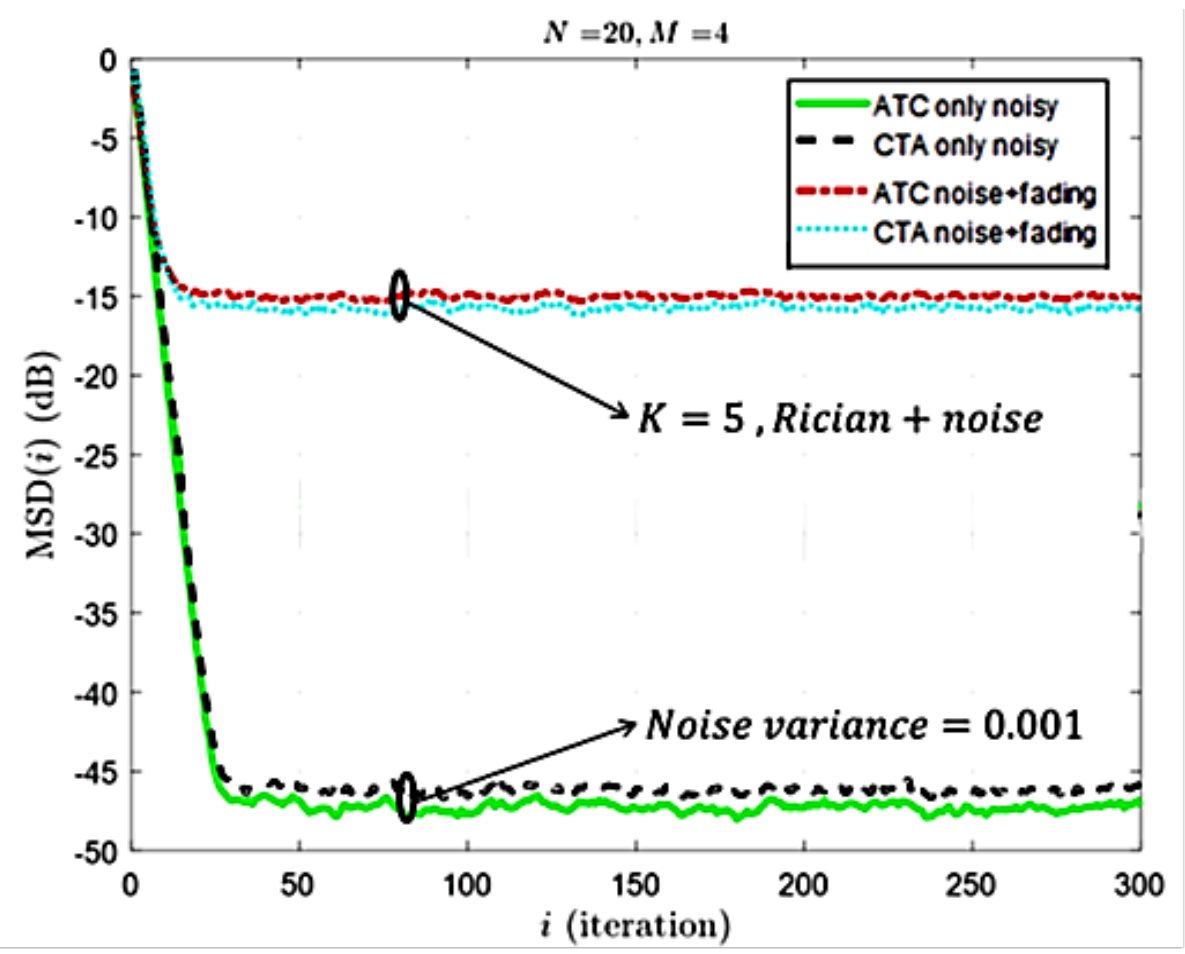

Fig. 6. Algorithms comparisons in noisy and noisy plus fading environments. 


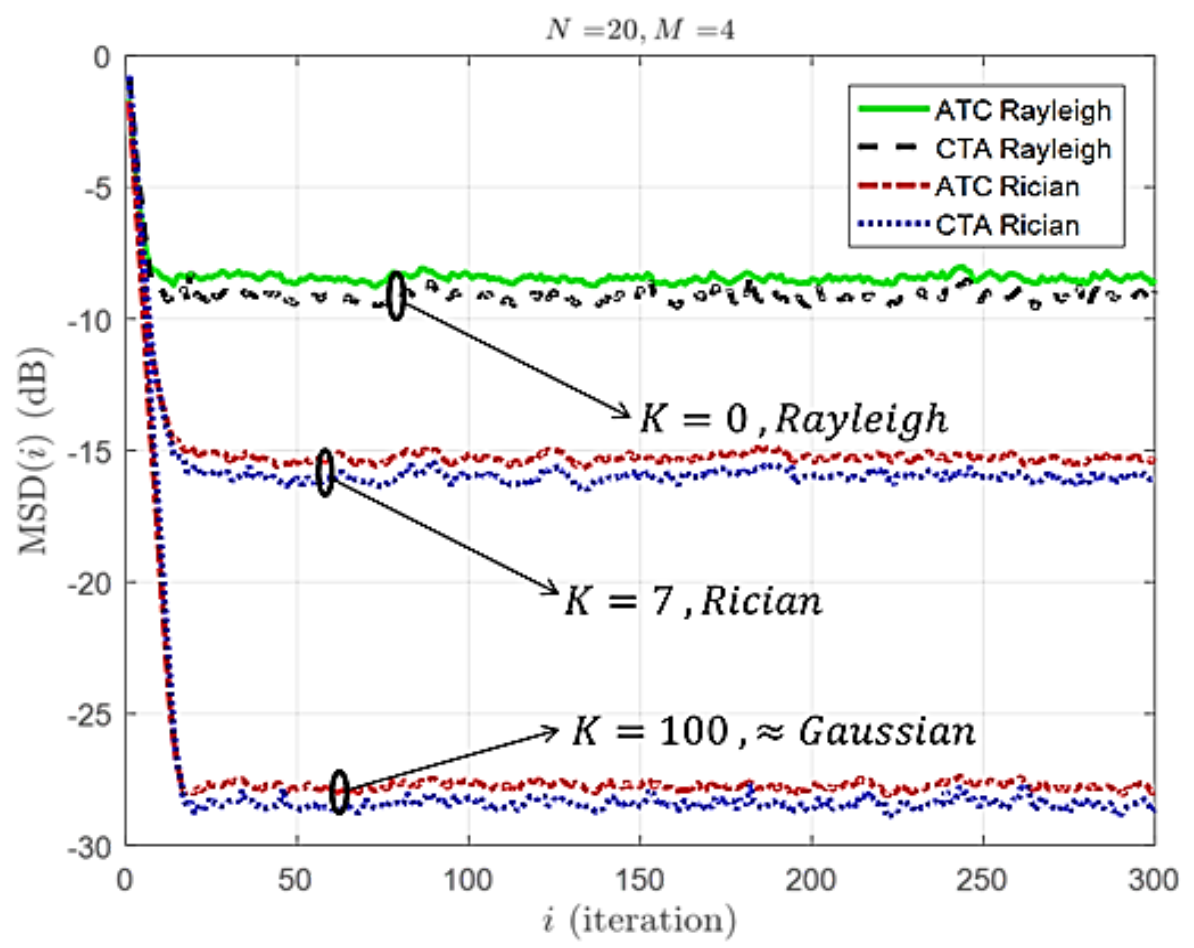

Fig. 7. Algorithms comparisons in noisy and noisy plus fading environments.

In Fig. 7, MSD curves are depicted for several cases of the Rician $K$ factor. We expected that the performance of the diffusion network relies on the value of $K$ and as the value of it increases, the power of the prominent path between nodes increase and consequently the performance of network gets better. We ran our simulations for 3 values of $K$ and presented the results for MSD of the network. It is important to mention when $K=0$, the fading is Rayleigh. In this figure, we can see that the performance of the network in Rician fading is much better than Rayleigh fading and as we mentioned earlier, the Rician fading model is a better assumption for adaptive networks than Rayleigh fading.

In order to show the MSD comparisons under different Rician factor $K$, we depict the MSD curves, where the performance of the diffusion network is presented for all rational values of $K$ factor at the same time.

In Fig. 8, we can see that as the value of $K$ factor increases, the performance diffusion network gets better. Also, in all the conditions the performance of CTA diffusion strategy is more acceptable than that of ATC diffusion scheme. 


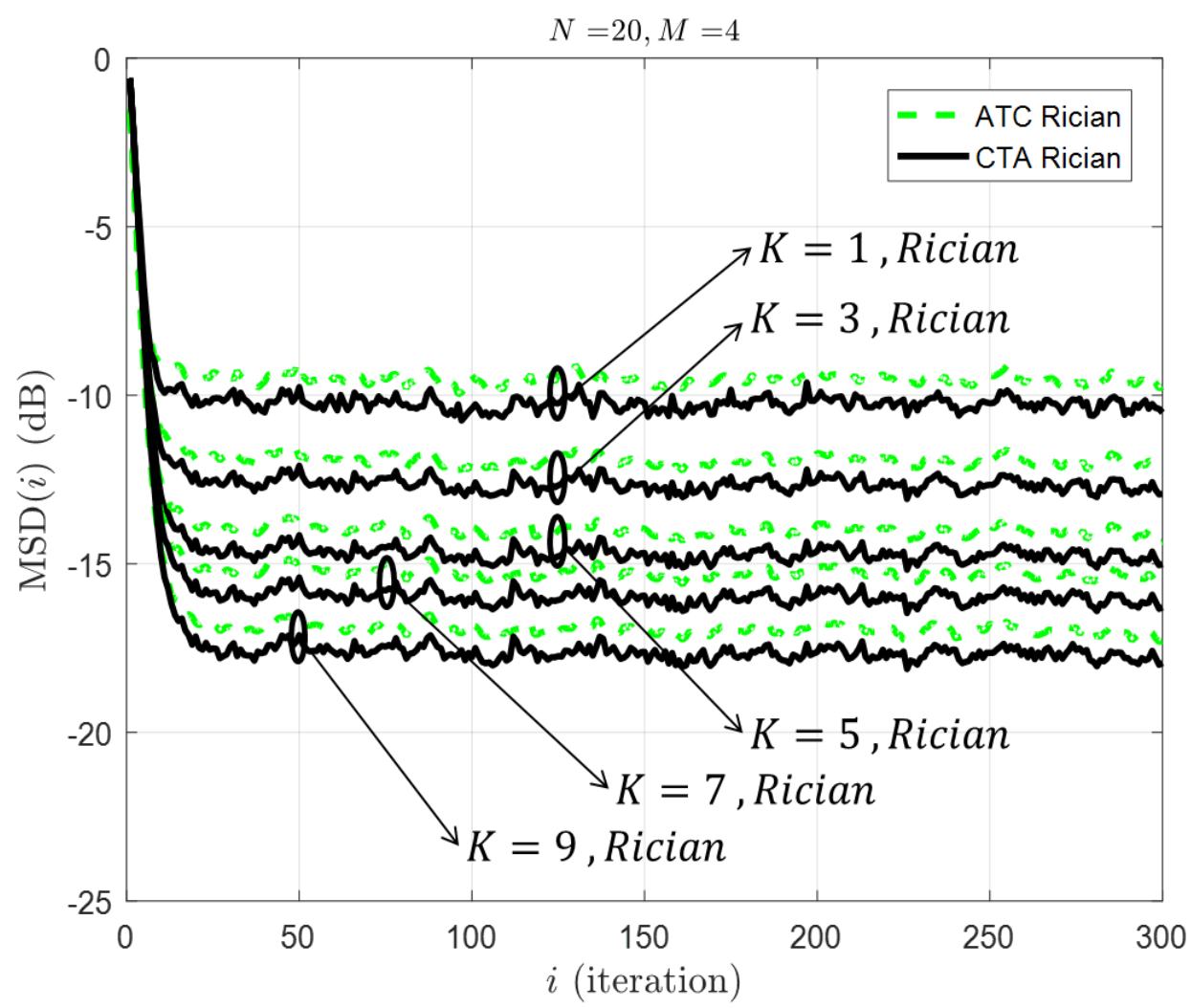

Fig. 8. Algorithm comparisons in Rician fading with different $K$ values.

\section{Conclusion}

In this paper, we investigated the performance of diffusion adaptive networks in the presence of Rayleigh and Rician fadings, respectively. First of all, we showed that the Rician fading for wireless adaptive networks is a more practical model than Rayleigh fading model. Secondly, we presented that the effect of fading is much more degrading for networks in comparison with noisy links. Finally, the computer simulation results demonstrated that in the presence of fading, the performance of the CTA diffusion strategy is more acceptable than ATC diffusion strategy. Other more advanced fading models can also be applied to the adaptive networks. In future works, we will work on the combining weights of diffusion strategy in order to find a suitable combination policy for reducing the effects of fading.

\section{Acknowledgements}

National Natural Science Foundation of China grants (No. 61401069), Jiangsu Specially Appointed Professor Grant (RK002STP16001), High-level talent startup grant of Nanjing University of Posts and Telecommunications (XK0010915026) and “1311 Talent Plan” of Nanjing University of Posts and Telecommunications. 


\section{References}

[1] Khalili A, Rastegarnia, A, Sanei S. "Performance analysis of incremental LMS over flat fading channels,” IEEE Trans. on control of network systems, 4(3), pp. 489 - 498, 2017. Article (CrossRef Link)

[2] R. Abdolee, B. Champagne and A. H. Sayed, "Diffusion LMS strategies for parameter estimation over fading wireless channels,” IEEE international conference on communications, pp. 19261930, 2013. Article (CrossRef Link)

[3] X. Zhao, S. Y. Tu, and A. H. Sayed, "Diffusion adaptation over networks under imperfect information exchange and non-stationary data,” IEEE Trans. signal Process. vol. 60, no. 7, pp. 3460-3475, 2012. Article (CrossRef Link)

[4] A. Khalili, A. Rastegarnia and W.M. Bazzi, "Effect of fading channels on the performance of distributed networks with cyclic cooperation,” ICTRC, Abu Dhabi, UAE, pp. 32-35, May 2015. Article (CrossRef Link)

[5] A. Rastegarnia, W.M. Bazzi, A. Khalili and J.A. Chambers, "Diffusion adaptive networks with imperfect communications: link failure and channel noise,” IET Signal Process., vol. 8, 59-66, 2014. Article (CrossRef Link)

[6] Wentao Ma, Jiandong Duan, Weishi Man, Junli Liang, Badong Chen, "General mixed-norm-based diffusion adaptive filtering algorithm for distributed estimation over network," IEEE Access, vol. 5, pp. 1090-1102, 2017. Article (CrossRef Link)

[7] A. H. Sayed and F. Cattivelli, "Distributed adaptive learning mechanisms," in Handb. Array Process. Sens. Networks, S. Haykin and K. J. Ray Liu, Eds. Wiley, 2009, pp. 693-722.

[8] J. Chen, C. Richard, A. H. Sayed, "Multitask diffusion adaptation over networks," IEEE Transactions on Signal Processing, vol. 62, no. 16, pp.4129-4144, Aug. 2014. Article (CrossRef Link)

[9] X. Zhao and A.H. Sayed, "Performance limits for distributed estimation over LMS adaptive networks,” IEEE Transactions on Signal Processing, vol. 60: 5107-5124, 2012. Article (CrossRef Link)

[10] J. Proakis and M. Salehi, Digital communications, 5th Ed. New York, NY, USA: McGraw-Hill, 2008.

[11] N. Kostov, “Mobile Radio Channels Modeling in MATLAB” Radioengineering Journal, vol. 12, no. 4, pp. 12 - 16, Dec. 2003. Article (CrossRef Link)

[12] Jianhua Zhang, Pan Tang, Lei Tian, Zixue Hu, Tan Wang, Wang Haiming, “6-100 GHz Research Progress and Challenges for Fifth Generation (5G) and Future Wireless Communication from Channel Perspective,” SCIENCE CHINA Information Sciences, vol. 60, no. 8, pp. 1-16, Aug. 2017. Article (CrossRef Link)

[13] Jianhua Zhang, "The Interdisciplinary Research of Big Data and Wireless Channel: A Cluster-Nuclei Based Channel Model”, China Communication, vol. 13, no. 2, pp. 14-26, 2016. Article (CrossRef Link)

[14] E. Mostafapour, C. Ghobadi, J. Nourinia, and M. Chehel Amirani, "Tracking performance of incremental LMS algorithm over adaptive distributed sensor networks,” Journal of communication engineering, 4, (1), 2015, pp. 1-10. Article (CrossRef Link)

[15] A. Khalili, M.A. Tinati, A. Rastegarnia, and J.A. Chambers, "Steady state analysis of diffusion LMS adaptive networks with noisy links,” IEEE Trans. Signal Process. vol. 60: 974-979, 2012. Article (CrossRef Link)

[16] W.M. Bazzi, A. Lotfza Pak, A. Rastegarnia, A. Khalili and Z. Yang, "Formulation and steady-state analysis of diffusion mobile adaptive networks with noisy links,” IET Signal Process., vol. 9: 631637, 2015. Article (CrossRef Link)

[17] A.H. Sayed, Adaptive Filters, New Jersey, NJ: Wiley, 2008.

[18] Wentao Ma, Badong Chen, et. al. "Sparse Least Logarithmic Absolute Difference Algorithm with Correntropy-Induced Metric Penalty,” Circuits Systems \& Signal Processing, vol. 35, no. 3, pp. 1077-1089, 2016. Article (CrossRef Link) 
[19] Li, Y., Y. Wang, and T. Jiang, "Sparse-aware set-membership NLMS algorithms and their application for sparse channel estimation and echo cancelation” AEÜ-International Journal of Electronics and Communications, vol. 70, no. 7, pp. 895-902, 2016. Article (CrossRef Link)

[20] Jianhua Zhang, Yuxiang Zhang, Yawei Yu, Ruijie Xu, Qingfang Zheng, Ping Zhang, "3D MIMO: How Much Does It Meet Our Expectation Observed from Channel Measurements?” IEEE Journal on Selected Areas in Communications, vol. 35, no. 8, pp. 1887 - 1903, Aug. 2017. Article (CrossRef Link)

[22] Y. Li, Y. Wang, and T. Jiang, "Norm-adaption penalized least mean square/fourth algorithm for sparse channel estimation,” Signal processing, vol.128, pp.243-251, 2016. Article (CrossRef Link)

[23] E. Mostafapour, A. Hoseini, J. Nourinia, and M. Chehel Amirani, "Channel estimation with adaptive incremental strategy over distributed sensor networks,” in Proc. IEEE $2^{\text {nd }}$ Int. Conf. on knowledge-based engineering and innovation, Tehran, Iran, 2015. Article (CrossRef Link)

[24] J. Chen, C. Richard, A. H. Sayed, "Diffusion LMS over multitask networks," IEEE Transactions on Signal Processing, vol. 63, no. 11, pp. 2733-2748, Jun. 2015. Article (CrossRef Link)

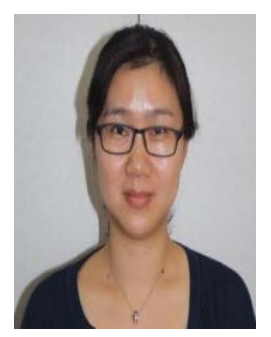

Jie Yang is a lecture in Nanjing University of Posts and Telecommunications, Nanjing, China. Her research interests are adaptive filter, compressive sensing, sparse dictionary designing, channel estimation, and advanced wireless techniques.

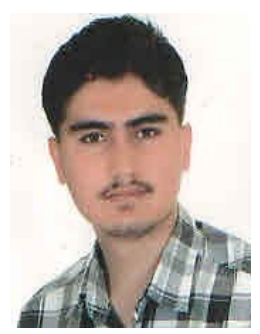

Ehsan Mostafapour was born in west Azarbayjan Province, Urmia, Iran, in 1988. He received the B.S. the M.S. degrees from the Islamic Azad University, in 2010 and 2012, respectively, both in telecommunication engineering. He also received his Ph.D. degree in January 2018 from the Department of Electrical Engineering, Urmia University, Urmia, Iran in telecommunication engineering. His research interests include stochastic and adaptive signal processing, wireless communications, adaptive networks and neural networks.

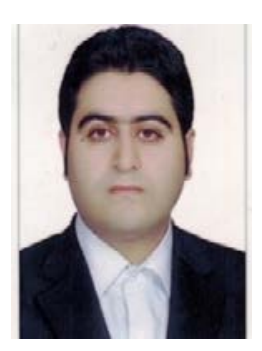

Amir Aminfar received his B.S. and M.S. degrees from the Islamic Azad University, in 2006 and 2009, respectively, both in electrical engineering. He also received his Ph.D. degree in September 2018 from the Department of Electrical Engineering, Urmia University, Urmia, Iran in telecommunication engineering. His research interests include adaptive networks, adaptive filters and the free space optical communication systems.

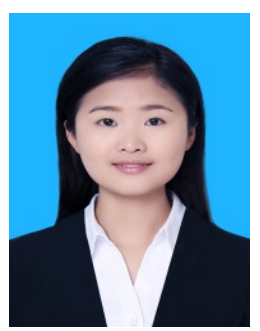

Jie Wang is a PhD candidate in Nanjing University of Posts and Telecommunications, Nanjing, China. Her research interests are adaptive filter, compressive sensing, sparse dictionary designing, channel estimation, and advanced wireless techniques. 

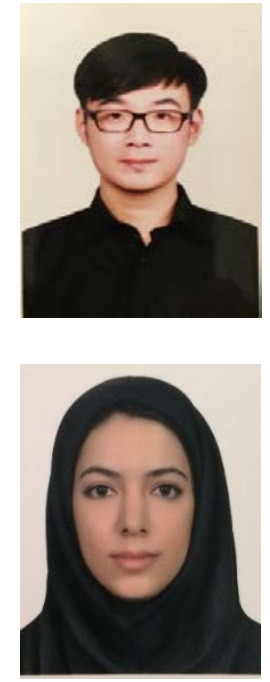

Afsaneh Akhbari received her B.Sc. degree in electrical engineering from the Imam Khomeini International University, Qazvin, Iran, in 2013, and the M.Sc. degree in electrical engineering from the Ghiaseddin Jamshid Kashani University, Abyek, Iran, in 2016. Her main research areas are adaptive filters, sparse signal processing and statistical Signal processing.

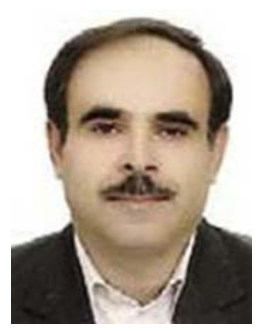

Changiz Ghobadi was born on 1 June, 1960 in Iran. He received the B.Sc. degree in Electrical and Electronic Engineering and M.Sc. degree in Electrical and Telecommunication Engineering from Isfahan University of Technology, Isfahan, Iran and Ph.D. degree in Electrical-Telecommunication from University of Bath, Bath, UK in 1998. From 1998 he was an Assistant Professor and now is full Professor in the Department of Electrical Engineering, of Urmia University, Urmia, Iran. His primary research interests are in antenna design, radar, and adaptive filters.

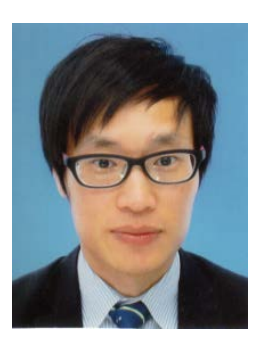

Guan Gui received the Dr. Eng degree in information and communication engineering from University of Electronic Science and Technology of China (UESTC), Chengdu, China, in 2011. From October 2009 to March 2012, with the financial supported from the China scholarship council (CSC) and the global center of education (ECOE) of Tohoku University, he joined the wireless signal processing and network laboratory (Prof. Adachi's laboratory), Department of Communications Engineering, Graduate School of Engineering, Tohoku University as for research assistant as well as postdoctoral research fellow,respectively. From September 2012 to March 2014, he was supported by Japan society for the promotion of science (JSPS) fellowship as postdoctoral research fellow at same laboratory. From April 2014 to October 2015, he was an Assistant Professor in Department of Electronics and Information System, Akita Prefectural University. Since November 2015, he has been a professor with Nanjing University of Posts and Telecommunications (NUPT), Nanjing, China. He is currently engaged in research of multidimensional system control, super-resolution radar imaging, adaptive filter, compressive sensing, sparse dictionary designing, channel estimation, and advanced wireless techniques. He is a member of Institute of Electrical and Electronics Engineers (IEEE).

Dr. Gui has been an Associate Editor for the Wiley Journal of Security and Communication Networks since 2012 and KSII Transactions on Internet and Information Systems since 2017. He received IEEE International Conference on Communications (ICC) Best Paper Award in years 2014 and 2017, as well as IEEE Vehicular Technology Conference (VTC-spring) Best Student Paper Award in year 2014. He was also selected as for Jiangsu Distinguished Professor and Jiangsu High-level Innovation and Entrepreneurial Talent in year 2016 and "1311 Talent Plan"Nanjing University of Posts and Telecommunications in year 2017. 